\title{
Genetic Polymorphism of NPHS1 Modifies the Clinical Manifestations of Ig A Nephropathy
}

\author{
Ichiei Narita, Shin Goto, Noriko Saito, Jin Song, Daisuke Kondo, Kentaro Omori, \\ Hiroshi Kawachi, Fujio Shimizu, Minoru Sakatsume, Mitsuhiro Ueno, and \\ Fumitake Gejyo
}

Division of Clinical Nephrology and Rheumatology (IN, SG, NS, JS, DK, KO, MS, MU, FG), and Department of Cell Biology (HK, FS), Institute of Nephrology, Niigata University Graduate School of Medical and Dental Sciences, Asahimachi-dori, Niigata, Japan

\begin{abstract}
SUMMARY: Nephrin, the molecule responsible for congenital nephrotic syndrome of Finnish type, is crucial in maintaining the glomerular filtration barrier. Recently, its complete gene structure and common gene polymorphisms in its exons have been reported, although the functional and clinical significance of these polymorphisms has not yet been elucidated. We investigated a possible association of the NPHS1 polymorphisms with the development of $\lg$ A nephropathy (IgAN), as well as the clinical and histologic manifestations in IgAN. A total of 464 Japanese subjects, including 267 patients with histologically proven IgAN and 197 healthy controls with normal urinalysis, were genotyped for the NPHS1 G349A, G2289A, and T3315C polymorphisms. The frequencies of the genotypes, alleles, and estimated haplotypes of NPHS1 polymorphisms were no different between patients with IgAN and the controls. Within the IgAN group, patients carrying at least one G allele of G349A tended to present with more proteinuria, lower renal function, and more severe histopathologic injury than those with the AA genotype, although the time from the first urinary abnormality to the renal biopsy was no different between both groups. The logistic regression analysis indicated that even after adjusting for the effect of proteinuria and hypertension the GG genotype of NPHS1 G349A was an independent risk factor for the deteriorated renal function at the time of diagnosis. This study suggests that the NPHS1 G349A polymorphism may be associated with heavy proteinuria and a decline in renal function in patients with IgAN. (Lab Invest 2003, 83:1193-1200).
\end{abstract}

\begin{abstract}
I A nephropathy (IgAN) is one of the major causes 1 of end-stage renal disease (ESRD) and the most common form of primary glomerulonephritis among patients undergoing renal biopsy throughout the world (Maisonneuve et al, 2000). It is characterized by mesangial proliferative glomerulonephritis with predominant deposits of IgA in the mesangial area. The actuarial renal survival in Japanese patients with IgAN at 10 years and 20 years is assumed to be $85 \%$ and $61 \%$, respectively, from the time when the first renal abnormalities are detected (Koyama et al, 1997). Familial clustering of IgAN and interindividual differences in the clinical course suggests that genetic factors may contribute to both the development and progression of this disease (Galla, 2001; Hsu et al, 2000). It has been well documented that impairment of renal function, severe proteinuria, and arterial hypertension
\end{abstract}

DOI: 10.1097/01.LAB.0000080600.49276.31

Received April 21, 2003.

This work was supported in part by health and labor science research grants for research on specific diseases from the Ministry of Health, Labour, and Welfare (to FG); and by a Grant-in-Aid for Scientific Research (No. 11671032) from the Ministry of Education, Culture, Sports, Science, and Technology of Japan (to IN).

Address reprint requests to: Dr. Ichiei Narita, Division of Clinical Nephrology and Rheumatology, Niigata University Graduate School of Medical and Dental Sciences, 757, Asahimachi-dori, Niigata, 951-8510, Japan. E-mail:naritai@med.niigata-u.ac.jp at the time of diagnosis are the strongest and most reliable clinical predictors of progression to ESRD (D'Amico, 2000; Koyama et al, 1997).

The nephrin gene, NPHS1 (AF035835), has recently been identified as the gene responsible for congenital nephrotic syndrome of the Finnish type (NPH1) (Kestila et al, 1998), an autosomal recessive disorder characterized by the onset of nephrotic syndrome, which usually occurs before 3 months of age (Hallman et al, 1956). NPH1 previously resulted in death before age 2 years, but it can now be treated by kidney transplantation, without the development of extrarenal symptoms (Holmberg et al, 1995). The NPHS1 gene consists of 29 exons spanning $26 \mathrm{~kb}$ in the chromosomal region 19q13.1 (Mannikko et al, 1995). In the kidney nephrin expression is observed only in visceral epithelial cells of the glomeruli, indicating its importance in the development or maintenance of the glomerular filtration barrier (Holthofer et al, 1999). In fact an mAb (mAb 5-1-6), which reacts with the slit diaphragm, induces a massive proteinuria (Orikasa et al, 1988), and the antigenic molecule recognized by mAb 5-1-6 has been identified as nephrin (Kawachi et al, 2000). The complete genomic structure for NPHS1 was reported, and a total of 50 mutations in the coding region of NPHS1 or the immediate 5'-flanking region have been identified in patients with NPH1 (Lenkkeri et al, 1999). In addition to these mutations, several sequence variants were published in Caucasian 
(Beltcheva et al, 2001), as well as in Japanese control individuals (Hirakawa et al, 2002) (an online database of Japanese single nucleotide polymorphisms [SNPs] can be found at http://snp.ims.u-tokyo.ac.jp). Although the functional significance of these gene polymorphisms remains unclear, it might be important to explore the phenotypic consequences imposed by the NPHS1 polymorphisms, not only in congenital nephrotic syndrome, but also in common glomerular diseases.

In this study to investigate the possible role of the NPHS1 polymorphism on the development of IgAN, as well as on the clinical manifestations of the disease, we performed a case control study for the NPHS1 G349A, G2289A, and T3315C polymorphisms in patients with IgAN and healthy controls in the Japanese population.

\section{Results}

In total we genotyped 464 subjects, which consisted of 267 patients with histologically proven IgAN and 197 healthy controls who were genotyped for NPHS1 G349A, G2289A, and T3315C. Table 1 lists the genotype distributions and the allele frequencies of these gene polymorphisms in both groups. The expected frequencies of the genotypes in both groups, under the assumption of Hardy-Weinberg equilibrium, did not differ from the observed genotype frequencies. No difference was recognized in the genotype and allele frequency between the patients with $\lg A N$ and the healthy controls without any history of renal disease or hypertension. Table 1 also shows estimated frequencies of four major haplotypes for the three SNPs loci in NPHS1. The frequencies of these haplotypes were no different between IgAN patients and controls. A complete linkage disequilibrium was observed between the loci 349 and 2289 ( $\left.D^{\prime}=1.0000, p<0.0001\right)$, but not between 3315 and the other two loci (349 and 3315, D' $=0.6513, p=0.0732 ; 2289$ and 3315, $D^{\prime}=$ $0.7480, p=0.1030)$.

Clinical characteristics of patients with $\lg A N$ at the time of renal biopsy are listed in Table 2. The comparisons shown in this table were made between patients with the homozygote of NPHS1 349A $(n=102)$ and those with either the heterozygote or homozygotes of 349G ( $n=165)$. There was no difference in the gender, age, body mass index, time from the first urine abnormality to renal biopsy, and blood pressure at the time of renal biopsy between the two groups. Urinary protein excretion at the time of renal biopsy in patients with the AA genotype tended to be less in comparison to patients with the other genotypes, but the difference was not statistically significant. The incidence of nephrotic range proteinuria (NS; urinary protein of

Table 1. Genotype Distributions and Allele Frequencies of Gene Polymorphisms in Patients with Histologically Proven IgAN and Healthy Controls

\begin{tabular}{|c|c|c|c|c|c|c|}
\hline & & & IgAN & Control & & \\
\hline \multirow[t]{10}{*}{ Genotype } & & & $N=267$ & $N=197$ & $\chi^{2}$ & $p$ Value \\
\hline & G349A & GG & 37 & 34 & & \\
\hline & & $\mathrm{GA}$ & 128 & 87 & & \\
\hline & & $\mathrm{AA}$ & 102 & 76 & 1.210 & 0.5460 \\
\hline & G2289A & GG & 178 & 143 & & \\
\hline & & GA & 82 & 51 & & \\
\hline & & AA & 7 & 3 & 2.130 & 0.3447 \\
\hline & T3315C & $\mathrm{TT}$ & 171 & 124 & & \\
\hline & & TC & 82 & 67 & & \\
\hline & & CC & 14 & 6 & 1.676 & 0.4326 \\
\hline \multirow[t]{7}{*}{ Allele } & & & $N=534$ & $N=394$ & & \\
\hline & G349A & G & 0.378 & 0.393 & & \\
\hline & & A & 0.622 & 0.607 & 0.219 & 0.638 \\
\hline & G2289A & G & 0.821 & 0.860 & & \\
\hline & & A & 0.179 & 0.140 & 2.687 & 0.101 \\
\hline & T3315C & $T$ & 0.793 & 0.802 & & \\
\hline & & C & 0.207 & 0.198 & 0.711 & 0.137 \\
\hline \multicolumn{7}{|c|}{ Haplotype estimated } \\
\hline G349A & G2289A & T3315C & $N=534$ & $N=394$ & & \\
\hline A & G & C & 0.136 & 0.156 & & \\
\hline A & G & $\mathrm{T}$ & 0.483 & 0.442 & & \\
\hline G & A & $\mathrm{T}$ & 0.145 & 0.146 & & \\
\hline G & G & $\mathrm{T}$ & 0.166 & 0.188 & & \\
\hline Others & & & 0.070 & 0.068 & 1.841 & 0.765 \\
\hline
\end{tabular}

IgAN, Ig A nephropathy. 


\begin{tabular}{|c|c|c|c|c|c|}
\hline & \multirow{2}{*}{$\begin{array}{l}\text { All patients } \\
N=267\end{array}$} & \multicolumn{2}{|c|}{ Genotype of NPHS1 G349A } & \multirow[b]{2}{*}{$p$ Value } & \multirow[b]{2}{*}{$\chi^{2}$} \\
\hline & & AA $N=102$ & GA/GG $N=165$ & & \\
\hline Gender (male \%) & 48.5 & 46.1 & 50.0 & NS & \\
\hline Age (year) & $37.1 \pm 13.4$ & $36.9 \pm 12.6$ & $37.2 \pm 13.8$ & NS & \\
\hline Body mass index & $22.56 \pm 3.20$ & $22.89 \pm 3.52$ & $22.34 \pm 3.69$ & NS & \\
\hline $\begin{array}{l}\text { Time from the first urine abnormality to renal } \\
\text { biopsy (month) }\end{array}$ & $57.6 \pm 67.7$ & $56.3 \pm 55.7$ & $58.4 \pm 74.5$ & NS & \\
\hline Urinary protein excretion (g/day) & $1.3 \pm 1.3$ & $1.1 \pm 0.9$ & $1.5 \pm 1.5$ & NS & \\
\hline Incidence of nephrotic range proteinuria (\%) & 7.9 & 2.0 & 11.5 & 0.0088 & 6.858 \\
\hline Serum creatinine $(\mathrm{mg} / \mathrm{dl})$ & $1.0 \pm 0.9$ & $1.0 \pm 1.3$ & $1.1 \pm 0.6$ & 0.0333 & \\
\hline Creatinine clearance (ml/min) & $87.5 \pm 32.3$ & $91.7 \pm 31.2$ & $84.9 \pm 32.8$ & 0.0223 & \\
\hline Incidence of advanced GN (\%) & 29.2 & 17.6 & 36.4 & 0.0012 & 10.477 \\
\hline \multicolumn{6}{|l|}{ Blood pressure $(\mathrm{mmHg})$} \\
\hline Systolic & $128.0 \pm 18.6$ & $129.1 \pm 19.5$ & $127.4 \pm 18.1$ & NS & \\
\hline Diastolic & $77.1 \pm 13.9$ & $78.4 \pm 14.1$ & $76.4 \pm 13.8$ & NS & \\
\hline Incidence of hypertension (\%) & 37.8 & 36.3 & 38.8 & NS & \\
\hline ACE-I or ARB administration (\%) & 8.6 & 9.8 & 7.9 & NS & \\
\hline
\end{tabular}

ACE-I, angiotensin-converting enzyme inhibitor; ARB, angiotensin-II receptor blocker; GN, glomerulonephritis.

more than $3.5 \mathrm{~g} /$ day) was significantly higher in patients with at least one G allele of the NPHS1 G349A polymorphism.

The level of serum creatinine at the time of renal biopsy was lower and the 24-hour creatinine clearance (Ccr) was higher in patients with the AA genotype than those with the GA or GG genotype. Moreover, the incidence of advanced glomerulonephritis (GN) was significantly higher in patients with the $G A$ or $G G$ genotypes of the G349A polymorphism than those with the AA genotype. Table 3 lists the frequencies of alleles for the NPHS1 polymorphisms in IgAN patients with or without NS, and those with or without advanced GN. Again, the G349A polymorphism was significantly associated with both NS and advanced GN. In contrast, T3315C was not associated with NS or advanced GN. In the haplotype analysis, in which haplotype frequencies for the G349A and G2289A loci were estimated in IgAN patients, the haplotype A-G was observed more frequently in the patients without NS and in those without advanced GN (Table 3). The T3315C polymorphism was not included in the haplotype estimation because this locus was not in linkage disequilibrium with the other two loci and was not associated with NS nor advanced GN.

The G349A in the NPHS1 were likely to be associated with both urinary protein excretion and renal function at the time of renal biopsy. However, these two clinical phenotypes were actually correlated to each other (data not shown). Therefore, to investigate whether the NPHS1 polymorphism affected the renal function at the time of renal biopsy independently of urinary protein excretion, the multivariate logistic regression analysis was used (Table 4). Because not all of the patients could be unequivocally assigned to have a particular haplotype and because the G349A

Table 3. Frequencies of Alleles for the NPHS1 Polymorphisms in Ig A Nephropathy Patients with or without Nephrotic Syndrome and Those with or without Advanced GN

\begin{tabular}{|c|c|c|c|c|c|c|c|c|c|}
\hline \multirow[b]{2}{*}{ SNP } & \multirow[b]{2}{*}{ Allele } & \multicolumn{2}{|c|}{ Nephrotic syndrome } & \multirow[b]{2}{*}{$\chi^{2}$} & \multirow[b]{2}{*}{$p$ Value } & \multicolumn{2}{|c|}{ Advanced GN } & \multirow[b]{2}{*}{$\chi^{2}$} & \multirow[b]{2}{*}{$p$ Value } \\
\hline & & $\begin{array}{c}\text { Yes } \\
N=42\end{array}$ & $\begin{array}{c}\text { No } \\
N=492\end{array}$ & & & $\begin{array}{c}\text { Yes } \\
N=156\end{array}$ & $\begin{array}{c}\text { No } \\
N=378\end{array}$ & & \\
\hline \multirow[t]{2}{*}{ G349A } & G & 0.524 & 0.366 & & & 0.474 & 0.339 & & \\
\hline & $A$ & 0.476 & 0.634 & 4.105 & 0.0428 & 0.526 & 0.661 & 8.651 & 0.0033 \\
\hline \multirow[t]{2}{*}{ G2289A } & $\mathrm{G}$ & 0.714 & 0.829 & & & 0.795 & 0.831 & & \\
\hline & $A$ & 0.286 & 0.171 & 3.470 & 0.0625 & 0.205 & 0.169 & 0.961 & 0.3270 \\
\hline \multirow[t]{2}{*}{ T3315C } & $\mathrm{T}$ & 0.738 & 0.799 & & & 0.769 & 0.804 & & \\
\hline & C & 0.262 & 0.201 & 0.871 & 0.3506 & 0.231 & 0.196 & 0.827 & 0.3631 \\
\hline \multicolumn{10}{|c|}{ Haplotype of G349A and G2289A } \\
\hline & $A-G$ & 0.476 & 0.634 & 4.105 & 0.0428 & 0.526 & 0.662 & 8.651 & 0.0033 \\
\hline & $\mathrm{G}-\mathrm{A}$ & 0.286 & 0.171 & 3.470 & 0.0625 & 0.208 & 0.169 & 0.961 & 0.3270 \\
\hline & $G-G$ & 0.238 & 0.195 & 0.449 & 0.5027 & 0.266 & 0.169 & 6.929 & 0.0085 \\
\hline
\end{tabular}

GN, glomerulonephritis; SNP, single nucleotide polymorphism. 
Table 4. Multiple Logistic Regression Analysis for a Deteriorated Renal Function at the Time Renal Biopsy

\begin{tabular}{lcccc}
\hline & $\chi^{2}$ & $p$ Value & Odds ratio & $95 \% \mathrm{Cl}$ \\
\hline Urinary protein excretion & & & & \\
$<1.0$ g/day & & & Referent & \\
1.0 to 3.5 g/day & 4.205 & 0.0403 & 1.969 & $1.030-3.763$ \\
$\quad>3.5$ g/day & 10.443 & 0.0012 & 7.315 & $2.188-24.456$ \\
Hypertension & & & Referent & \\
No & 13.677 & 0.0002 & 3.194 & $1.726-5.911$ \\
Yes & & & & \\
Genotype of NPHS1 G349A & & & Referent & \\
AA & 3.560 & 0.0452 & 1.982 & $1.032-4.033$ \\
AG & 6.341 & 0.0118 & 3.299 & $1.303-8.354$ \\
GG & & &
\end{tabular}

polymorphism was most significantly associated with a deteriorated renal function, the G349A genotype was included in the multivariate analysis. In this analysis, the NPHS1 G349A genotype was found to be an independent risk factor for advanced GN at the time of renal biopsy after adjusting for the effect of urinary protein excretion and hypertension. The odds ratios for the deteriorated renal function in patients with the $A G$ or $G G$ genotype versus those with $A A$ as the reference were 1.982 (95\% confidence interval, 1.032 to $\left.4.033, \chi^{2}=3.560, p=0.0452\right)$ and $3.299(95 \%$ confidence interval, 1.303 to $8.354, \chi^{2}=6.341, p=$ $0.0118)$, respectively.

Next, any possible associations between the NPHS genotype and histopathologic findings were investigated. Figure 1 shows the mean values of each histopathologic grading score in patients with the $A A$ and AG/GG genotypes of NPHS1 G349A. Patients carrying at least one $\mathrm{G}$ allele of the NPHS1 G349A polymorphism had more severe histopathologic dam-
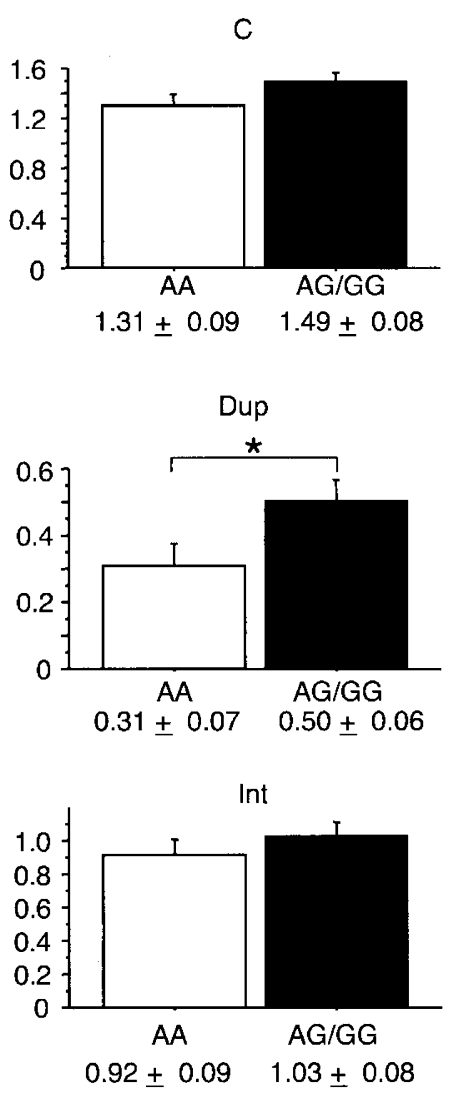

M

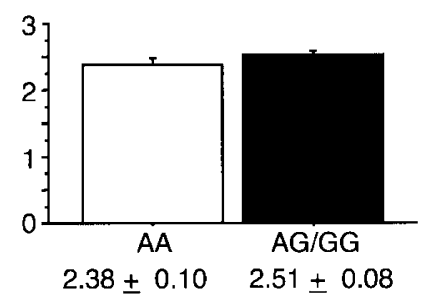

Cres

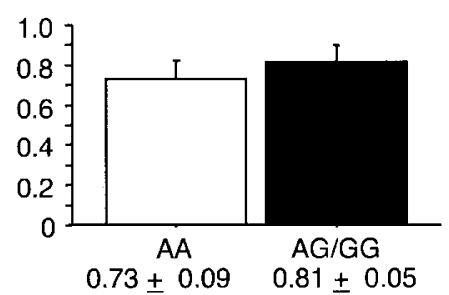

Endo

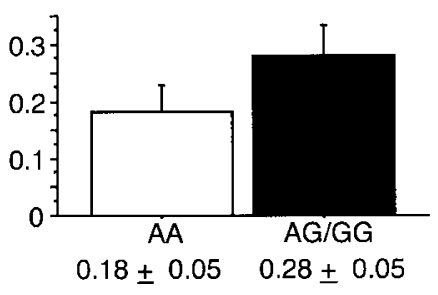

$\mathrm{Ad}$

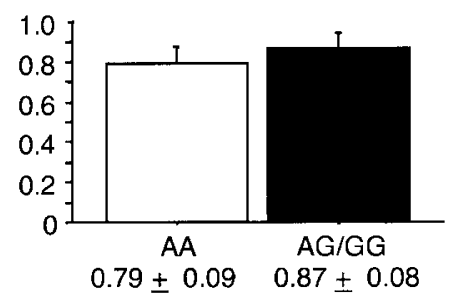

Figure 1.

Mean values of each histopathologic grading score in Ig A nephropathy patients with the AA ( $\square$ ) and AG/GG ( $\square$ ) genotypes of the NPHS1 G349A polymorphism. Glomerular changes were scored for each glomerulus, and the average score of each was calculated. The scores for mesangial cell proliferation ( $C$ ) and mesangial matrix increase $(M)$ were graded into five grades ranging from 0 to 4 . Other glomerular changes including endocapillary proliferation (Endo), duplication of glomerular basement membrane (Dup), crescent formation (Cres), and adhesion of tufts to Bowman's capsule (Ad), as well as tubulointerstitial lesions (Int), were graded 0 to 4 according to their incidence. Data are given as mean $\pm \mathrm{SE},{ }^{\star} p<0.05$ by Mann-Whitney $U$ test. 
age, in particular, with respect to duplication of the glomerular basement membrane (AG/GG versus $A A$; $0.51 \pm 0.70$ versus $0.31 \pm 0.58, p=0.0307$, by Mann-Whitney $U$ test). No significant deference was observed between patients with the $A A$ and $A G / G G$ genotype in mean values of other histologic scores including mesangial cell proliferation, matrix increase, interstitial fibrosis, adhesion of glomerular tufts to Bowman's capsule, crescent formation, and endocapillary proliferation. However, patients with AG/GG genotype had a tendency to present with numerically higher grades in all respects to the histologic scores evaluated.

\section{Discussion}

The results of the present study indicate a role of the genetic polymorphism of NPHS1, not in the development, but in the clinical manifestations of IgAN. The IgAN patients with the AG/GG genotypes of NPHS1 G349A presented with more proteinuria, as well as an increased deterioration in renal function compared with those with AA genotypes. The results of the histopathologic assessments of the patients were in accordance with the clinical data. T3315C, which is 20-kb distant from G349A and 13kb from G2289A, was neither in linkage disequilibrium with the other two loci, nor associated with these clinical phenotypes. Although G349A and G2289A were in tight linkage disequilibrium, the significance of the association with heavy proteinuria and a deteriorated renal function was higher in G349A than in G2289A. This is thought to be due to the difference in allele frequency of these two SNPs, because it has been reported that in association studies SNPs with high allele frequencies are more statistically informative than those with low allele frequencies (Martin et al, 2000). In the haplotype analysis, the A-G haplotype was found to be a protective haplotype both for NS and for advanced GN. These clinical phenotypes are well known as significant risk factors for the progression to ESRD. However, to establish the prognostic significance of this gene polymorphism, a prospective randomized controlled study with a long-term observation is needed.

The filtration barrier of the glomerulus consists of a fenestrated endothelium, a layered glomerular basement membrane, and visceral epithelial cells, podocytes. Accumulating evidence indicates that the podocytes, with their primary and secondary foot processes, as well as the interpodocyte slit membranes, are the final glomerular barrier, which prevents the leakage of circulating macromolecules. Nephrin is a major molecule in the podocyte filtration slits. The remarkable regulation of nephrin-specific mRNA levels in experimental models has been reported (Luimula et al, 2000). More recently, a typical down-regulation of nephrin expression has been reported in the glomeruli of samples from patients with IgAN, membranous nephropathy, and membranoproliferative glomerulonephritis (Aaltonen et al, 2001; Kim et al, 2002; Wang et al, 2002). The predicted protein product of the NPHS1 is a transmembrane protein with eight Ig-like extracellular repeats and a uniquely structured intracellular domain (Kestila et al, 1998). The SNP G349A exists in exon three of the nephrin genes and is accompanied by the substitution of amino acid Lys for Glu117, whereas the other two SNPs do not cause amino acid substitution (Lenkkeri et al, 1999). Considering the pivotal role of the molecule in maintaining glomerular permselectivity, it is not surprising that the polymorphism has an association with urinary protein excretion and the incidence of heavy proteinuria caused by glomerular injury. Although, to date, there is no evidence available on the functional significance of the NPHS1 polymorphism, an alteration in the function of the nephrin molecule, even to a minor extent, would lead to an alteration in both the physiologic and pathologic states of the glomerular podocyte. This in turn may affect the response of intrinsic renal cells to immunological injury such as IgAN, resulting in the clinical phenotype, whereas it would have no effect on the clinical phenotype in normal physiological conditions. There is also the possibility that other gene polymorphism, which is in linkage disequilibrium with the G349A in NPHS1, has a direct impact on expression or function of the gene and is responsible for the association observed in this study. We cannot provide the molecular mechanism by which the NPHS1 polymorphism affects the renal function, as well as the urinary protein excretion. It might be argued that the association between the NPHS1 polymorphism and the deteriorated renal function observed at the time of renal biopsy was a indirect consequence of the heavier proteinuria in patients with a $349 \mathrm{G}$ allele of the NPHS1 polymorphism, because proteinuria is well known as a factor for renal damage. However, the result of the logistic regression analysis indicated the effect of the SNP on renal function was independent to proteinuria. This may suggest that nephrin plays an important role not only in maintaining the glomerular filtration barrier, but also in regulating podocyte function and its cell behavior in the disease state. It is crucial to clarify the functional significance of this genetic polymorphism especially on the podocyte function both in the normal physiological condition and in the inflammatory response.

Our recent investigation has demonstrated that podocyte injury accompanied by down-regulation of nephrin is one of the important factors in bringing on irreversible glomerular alterations (Morioka et al, 2001). It is possible that an alteration in the expression of nephrin in the pathologic state, which might be associated with the NPHS1 polymorphism, is followed by more severe glomerular injury. The limitation of the present study is that we could not clarify the mechanism by which the NPHS1 was associated with severe histologic injury and that we could not provide direct evidence for an association between the NPHS1 polymorphism and the expression of nephrin protein in patients with IgAN. However, the histologic investigation in this study may support the possibility that the NPHS1 polymorphism has a direct or specific effect on podocyte in the disease state. 
We selected IgAN patients as the subjects of this investigation because they are the largest population of patients with an identical histologic disease entity. Moreover, the patients with IgAN in this study were diagnosed at a single center, and the records of their clinical data at the time of renal biopsy were available. The effect of the genetic polymorphisms in NPHS1 on the clinical manifestations of glomerular disease observed in this study may not be specific to IgAN. It is rather likely that the gene polymorphism investigated in this study affects the clinical phenotypes in other types of glomerular diseases. Although, if no association is found with other disease groups, that may provide an important negative control. However, it is thought that small sample size groups with heterogeneous disease entities are inadequate for proving any negative or positive association between genotype and phenotype.

There is a possibility that the NPHS1 polymorphism merely provides a fortunate marker of other differences in the genetic background that serves as genetic modifiers. A further large population-based and genome-wide study is necessary to clarify the genetic factors contributing to the development of $\lg \mathrm{AN}$, and this would be helpful in understanding the pathogenetic mechanism of IgAN as well as developing a new strategy for treatment of the disease.

Although further study is necessary to elucidate the functional significance of the NPHS1 polymorphism on nephrin expression or podocyte function, the findings of this study support the important role of podocyte damage for developing and progression of glomerular injury in IgAN.

\section{Materials and Methods}

\section{Patients}

The protocol for the genetic study was approved by the ethics committee of the institution involved, and informed written consent for the genetic studies was obtained from all participants.

Genomic DNA of peripheral blood cells from 464 Japanese individuals including 267 patients with histologically confirmed IgAN was isolated using an automatic DNA isolation system (NA-1000, Kurabo, Osaka, Japan). Schönlein-Henoch purpura and secondary IgAN such as hepatic glomerulosclerosis were excluded from the analysis. Diagnosis of IgAN in all cases was based on a kidney biopsy that revealed the presence of dominant or co-dominant glomerular mesangial deposits of IgA as assessed by an immunofluorescence examination. The mean age at diagnosis was $37.1 \pm 13.4$ (range from 9 to 74 ) years.

Clinical characteristics of the patients with IgAN, including gender, age, body mass index, duration of observation (in months), level of urinary protein excretion (g/day), serum creatinine $(\mathrm{mg} / \mathrm{dL})$, and $\mathrm{Ccr}(\mathrm{ml} /$ min), were investigated before the start of any treatment. The time from the first urine abnormality to renal biopsy (month) was also recorded for 213 of the 267 patients, where the first episode of urine abnormality (proteinuria or hematuria) could be clearly defined. Advanced GN was defined by a 24-hour CCr of $<70$ $\mathrm{ml} / \mathrm{min} / 1.73 \mathrm{~m}^{2}$ body surface area. Hypertension was defined by the use of one or more antihypertensive medications and/or a blood pressure greater than or equal to $140 \mathrm{mmHg}$ systolic or $90 \mathrm{mmHg}$ diastolic. Only 23 of the 267 (8.6\%) patients were administrated angiotensin-converting enzyme inhibitors and/or angiotensin receptor antagonists before the diagnosis.

To provide a control for the local genotype frequency being examined, 197 Japanese volunteers (98 female and 99 male) with no history of renal disease or hypertension and with normal urinalysis were also recruited. The mean age of healthy controls was 39.2 \pm 10.6 years, and this was no different from that of the patients $(p=0.0817)$.

\section{Determination of the Genotypes}

After investigating the clinical characteristics from past medical records, genotypes for the NPHS1 G349A, G2289A, and T3315C polymorphisms, which are located in exon 3,17 , and 25 of the gene, respectively, were independently determined in a double-blind fashion by allele specific oligonucleotide hybridization after PCR (PCR) using allele specific primers as shown in Table 5. These SNPs were selected for analysis because the frequencies of their minor alleles were more than 0.1 in a preliminary analysis, in which 100 healthy controls were genotyped. A DNA fragment containing the SNP region of the NPHS1 was amplified by PCR, with two allelespecific sense primers and biotin-labeled antisense primers or vice versa, which were designed based on the published sequence (GenBank accession number AF035835). The second base from the 3 '-end of the allele specific primer had each allele specific sequence, and the third base had an artificial mismatch sequence. The artificial mismatch was a mixture of nucleotides $\mathrm{G}$ or $\mathrm{C}$ to obtain the highest specificity in the following hybridization reaction. The reaction mixture in KOD plus buffer $(25 \mu \mathrm{l})$ contained $0.02 \mu \mathrm{g}$ of DNA, $5 \mathrm{pmol}$ of each oligonucleotide primer, $0.2 \mathrm{~mm}$ each deoxynucleoside triphosphate, $0.9 \mathrm{~mm} \mathrm{MgSO}_{4}$, and 1 unit of DNA polymerase (KOD plus, Toyobo, Osaka, Japan). The amplification protocol comprised of an initial denaturation at $94^{\circ} \mathrm{C}$ for 5 minutes; 35 cycles of denaturation at $95^{\circ} \mathrm{C}$ for 15 seconds, annealing at $65^{\circ} \mathrm{C}$ for 30 seconds, and an extension at $68^{\circ} \mathrm{C}$ for 30 seconds, with a final extension at $68^{\circ} \mathrm{C}$ for 2 minutes. Amplified DNA was denatured with $\mathrm{NaOH}$, and then hybridized at $37^{\circ} \mathrm{C}$ for 30 minutes in hybridization buffer containing $30 \%$ formamide with allele-specific capture probes fixed to the bottom of the wells of a 96-well plate (Table 5). The wells were then washed thoroughly, and alkaline phosphataseconjugated streptoavidin added to each, before the plate was incubated at $37^{\circ} \mathrm{C}$ for 15 minutes. After washing each well thoroughly, $0.8 \mathrm{~mm}$ WST-1 (2-(4iodophenyl)-3-(4-nitrophenyl)-5-(2,4-disulfophenyl)$2 \mathrm{H}$-tetrazolium, monosodium salt) and $0.4 \mathrm{~mm}$ BCIP (5-bromo-4-chloro-3-indolyl phosphate p-toluidine 
Table 5. The Nucleotide Sequences of Primers for Allele Specific PCR and Hybridization Probes

\begin{tabular}{|c|c|c|}
\hline SNP & & \\
\hline \multirow[t]{5}{*}{ G349A } & Forward primer & 5'-CCAGGTCGGCCGCTCXGA-3' \\
\hline & & 5'-CCAGGTCGGCCGCTCXAA-3' \\
\hline & Reverse primer & 5'-biotin-CCATACCCAGGATGGAGAGGATCAC-3' \\
\hline & Hybridization probes & 5'-CCGCTCXGAGATGGGGCC-3 \\
\hline & & 5'-CCGCTCXAAGATGGGGCCCT-3' \\
\hline \multirow[t]{5}{*}{ G2289A } & Forward primer & 5'-biotin-TGGATCCTCACCAGTCTCTCC-3' \\
\hline & Reverse primer & 5'-TGTGGACATAGTCTGCACTGXCG-3' \\
\hline & & 5'-TGTGGACATAGTCTGCACTGXTG-3' \\
\hline & Hybridization probes & 5'-TCTGCACTGXCGATGCCAAT-3' \\
\hline & & 5'-TCTGCACTGXTGATGCCAAT-3' \\
\hline \multirow[t]{5}{*}{ T3315C } & Forward primer & 5'-biotin-TGGCTCTCCTCATATTCGTTC-3' \\
\hline & Reverse primer & 5'-CCTTCATCCTGGAAGGTXAG-3' \\
\hline & & 5'-CTTCATCCTGGAAGGTXGG-3' \\
\hline & Hybridization probes & 5'-CTGGAAGGTXAGAAGAGGACC-3' \\
\hline & & 5'-CTGGAAGGTXGGAAGAGGACC-3' \\
\hline
\end{tabular}

SNP, single nucleotide polymorphism.

salt), a substrate for alkaline phosphatase, were added and the colorimetry was measured.

To confirm the accuracy of the genotyping method, we also performed direct sequencing of the PCR products from 48 individuals (24 lgAN patients and 24 healthy controls randomly selected) using the TaqDNA polymerase cycle sequencing method. A BigDye Terminator Cycle Sequencing FS Kit was used according to the manufacturer's instruction (Perkin-Elmer, Foster City, California). The pairs of oligonucleotide primers as described previously were used for the bidirectional sequencing (Lenkkeri et al, 1999). An automated DNA sequencer (model ABI PRISM 310, Perkin-Elmer) was used for the analysis. The results of the direct sequencing were completely consistent with those of the allele-specific PCR method described above.

\section{Histopathological Analysis}

Histopathologic findings of kidney biopsy specimens of IgAN patients were classified according to the classification described previously (Suzuki et al, 1992). A single pathologist evaluated all specimens by light microscopy in a double-blind fashion. Glomerular changes were scored for each glomerulus, and the average score of each was calculated. The scores of cellular proliferation and the matrix increase in the mesangium were graded into five grades ranging from 0 (minimal change) to 4 (diffuse global marked). Other glomerular changes including endocapillary proliferation, duplication of glomerular basement membrane, crescent formation, and adhesion of tufts to Bowman's capsule as well as tubulointerstitial lesions were graded 0 to 4 according to their incidence. Grades 0 to 4 represent an incidence of the lesion in $0 \%$ to $4 \%$, $5 \%$ to $24 \%, 25 \%$ to $49 \%, 50 \%$ to $74 \%$, and $75 \%$ to $100 \%$ of cases, respectively.

\section{Statistical Analysis}

Haplotype frequencies for sets of alleles were estimated using ARLEQUIN software version 2.0, which was based on a maximum-likelihood method. (Genetics and Biometry Laboratory, Department of Anthropology, University of Geneva, Geneva, Switzerland; http://lgb.unige.ch/arlequin/). Pair-wise linkage disequilibrium coefficients ( $\left.D^{\prime}\right)$ were also calculated using ARLEQUIN version 2.0 and expressed as the $D^{\prime}=$ D/D max, according to Slatkin (Slatkin, 1994).

Statview 5.0 statistical software (Abacus Concepts, Inc., Berkeley, California) was used for statistical analyses on a Macintosh G4 computer. Chi-square analysis was used when comparing allele frequencies and categorical variables between the groups. HardyWeinberg equilibrium was tested by a Chi-square test with $1 \mathrm{df}$. The adjusted odds ratios and 95\% confidence interval for advanced GN at the time of renal biopsy with multivariate factors were calculated using logistic regression analysis. Variables that achieved statistical significance $(p<0.05)$ in the univariate analysis were subsequently included in a multivariate analysis using a stepwise forward logistic regression procedure and the effects of these covariates were expressed by an odds ratio. A $p$ value of $<0.05$ was considered statistically significant.

\section{Acknowledgements}

A part of this study was presented at the 2002 American Society of Nephrology Renal Week, Philadelphia, Pennsylvania, and was published in abstract form (J Am Soc Nephrol 13:519A, 2002). The authors wish to thank Naofumi Imai, Keiko Yamagiwa, Noriko Ikeda, and Kumiko Furui for excellent technical assistance.

\section{References}

Aaltonen P, Luimula P, Astrom E, Palmen T, Gronholm T, Palojoki E, Jaakkola I, Ahola H, Tikkanen I, and Holthofer $\mathrm{H}$ (2001). Changes in the expression of nephrin gene and protein in experimental diabetic nephropathy. Lab Invest 81:1185-1190. 
Beltcheva O, Martin P, Lenkkeri U, and Tryggvason K (2001). Mutation spectrum in the nephrin gene (NPHS1) in congenital nephrotic syndrome. Hum Mutat 17:368-373.

D'Amico G (2000). Natural history of idiopathic IgA nephropathy: Role of clinical and histological prognostic factors. Am J Kidney Dis 36:227-237.

Galla JH (2001). Molecular genetics in IgA nephropathy. Nephron 88:107-112.

Hallman N, Hjelt L, and Ahvenainen EK (1956). Nephrotic syndrome in newborn and young infants. Ann Peadiatr Fenn 2:227-241.

Hirakawa M, Tanaka T, Hashimoto Y, Kuroda M, Takagi T, and Nakamura Y (2002). JSNP: A database of common gene variations in the Japanese population. Nucleic Acids Res 30:158-162.

Holmberg C, Antikainen M, Ronnholm K, Ala Houhala M, and Jalanko H (1995). Management of congenital nephrotic syndrome of the Finnish type. Pediatr Nephrol 9:87-93.

Holthofer H, Ahola H, Solin ML, Wang S, Palmen T, Luimula P, Miettinen A, and Kerjaschki D (1999). Nephrin localizes at the podocyte filtration slit area and is characteristically spliced in the human kidney. Am J Pathol 155:1681-1687.

Hsu SI, Ramirez SB, Winn MP, Bonventre JV, and Owen WF (2000). Evidence for genetic factors in the development and progression of IgA nephropathy. Kidney Int 57:1818-1835.

Kawachi H, Koike H, Kurihara H, Yaoita E, Orikasa M, Shia MA, Sakai T, Yamamoto T, Salant DJ, and Shimizu F (2000). Cloning of rat nephrin: Expression in developing glomeruli and in proteinuric states. Kidney Int 57:1949-1961.

Kestila M, Lenkkeri U, Mannikko M, Lamerdin J, McCready P, Putaala H, Ruotsalainen V, Morita T, Nissinen M, Herva R, Kashtan CE, Peltonen L, Holmberg C, Olsen A, and Tryggvason K (1998). Positionally cloned gene for a novel glomerular protein-nephrin-is mutated in congenital nephrotic syndrome. Mol Cell 1:575-582.

Kim BK, Hong HK, Kim JH, and Lee HS (2002). Differential expression of nephrin in acquired human proteinuric diseases. Am J Kidney Dis 40:964-973.

Koyama A, Igarashi M, and Kobayashi M (1997). Natural history and risk factors for immunoglobulin A nephropathy in Japan. Research Group on Progressive Renal Diseases. Am J Kidney Dis 29:526-532.

Lenkkeri U, Mannikko M, McCready P, Lamerdin J, Gribouval O, Niaudet PM, Antignac CK, Kashtan CE, Homberg C, Olsen A, Kestila M, and Tryggvason K (1999). Structure of the gene for congenital nephrotic syndrome of the Finnish type (NPHS1) and characterization of mutations. Am J Hum Genet 64:51-61.
Luimula P, Ahola H, Wang SX, Solin ML, Aaltonen P, Tikkanen I, Kerjaschki D, and Holthofer H (2000). Nephrin in experimental glomerular disease. Kidney Int 58:1461-1468.

Maisonneuve P, Agodoa L, Gellert R, Stewart JH, Buccianti G, Lowenfels AB, Wolfe RA, Jones E, Disney AP, Briggs D, McCredie M, and Boyle P (2000). Distribution of primary renal diseases leading to end-stage renal failure in the United States, Europe, and Australia/New Zealand: Results from an international comparative study. Am J Kidney Dis 35:157165.

Mannikko M, Kestaila M, Holmberg C, Norio R, Ryynanen M, Olsen A, Peltonen L, and Tryggvason K (1995). Fine mapping and haplotype analysis of the locus for congenital nephrotic syndrome on chromosome 19q13.1. Am J Hum Genet 57: 1377-1383.

Martin ER, Lai EH, Gilbert JR, Rogala AR, Afshari AJ, Riley J, Finch KL, Stevens JF, Livak KJ, Slotterbeck BD, Slifer SH, Warren LL, Conneally PM, Schmechel DE, Purvis I, PericakVance MA, Roses AD, and Vance JM (2000). SNPing away at complex diseases: Analysis of single-nucleotide polymorphisms around APOE in Alzheimer disease. Am J Hum Genet 67:383-394

Morioka Y, Koike H, Ikezumi Y, Ito Y, Oyanagi A, Gejyo F, Shimizu F, and Kawachi H (2001). Podocyte injuries exacerbate mesangial proliferative glomerulonephritis. Kidney Int 60:2192-2204.

Orikasa M, Matsui K, Oite T, and Shimizu F (1988). Massive proteinuria induced in rats by a single intravenous injection of a monoclonal antibody. J Immunol 141:807-814.

Slatkin M (1994). Linkage disequilibrium in growing and stable populations. Genetics 137:331-336.

Suzuki S, Sato H, Kobayashi H, Takayama R, Maruyama $\mathrm{Y}$, Ogino $\mathrm{S}$, Ueno $\mathrm{H}$, Inomata $\mathrm{A}$, Nishi $\mathrm{S}$, Saito $\mathrm{T}$, and et al (1992). Comparative study of IgA nephropathy with acute and insidious onset. Clinical, laboratory and pathological findings. Am J Nephrol 12:22-28.

Wang SX, Rastaldi MP, Patari A, Ahola H, Heikkila E, and Holthofer H (2002). Patterns of nephrin and a new proteinuria-associated protein expression in human renal diseases. Kidney Int 61:141-147. 